lent. The former usually ends in resulution, and the latter yields rcadily to judicious treatment.

Any one desiring further and especially meteorological data is respectfully referred to my contribution to the last census, made at the request of the department.

San Diego, March ro, 1886.

\section{ERYSIPELATOUS ECZEMA.}

IBY GLO. N. MUNLITIS, M.D., Or NEW ORLRANS, LA.

I have observed, during my past year's experience, a great number of cases of an exanthematous eruption, which, from the prodromal phenomena and the subsecjuent ultimate pathological phenomena, I am compelled to denominate "erysipelatous ecema." As an initiative, a rigor, with features of a pyretic nature, vertigo slight, a tongue slightly coated, with a metallic taste, some pains, arthritic in locality, urine somewhat highly-colored, pulse somewhat excited and full, consonant with the succeeding turgescence of the cutaneous surface.

The primary features, naturally, so greatly resembling scarlatina, woukd lead one to diagnosticate a case of the latter named malady, yet upon close observation, it develops into an acute form of dermatitis. 'There were other features indicating an attack of erysipelas pure, in its primary manifestation, yet there were no bulle, and no apparent anasarcous condition of the integument, as in erysipelas, but with a vigorously congestive and hypertrophied condition, sometimes corrugated and corded like elephantiasis. Itching was an intolerable accompaniment, yet no wheals were present, as in urticaria, no exudative plastic fluid, as in eczema, and no exfoliation as profuse as in ecrema chronicus.

Dermatitis acutus would be a suitable synonym, yet owing to the characteristic features, I think the compound term more expressive. The duration of the malady was from one to three weeks, unlike erysipelas, running its prescribed course within five days, with no unusual developments, as one might apprehend from such an universal congestion, so to speak, of the whole cutaneous surface, with peripheral paresis of neurosal filaments. The last case, as I observed it, presented a pachydermatous condition with ruga and some exfoliative desquamation, disfiguring the face of the old lady considerably. Her general health was and had always been good, and there were no extraneous concomitants to arouse any suspicion of infection. The features present were identical with those seen previously.

Now as to the causes. I did observe some years since that several cooks whom I had treated developed bona fide eczema of the face, neck and forearm. I can recall some of washerwomen who suffered with an eruption on the hands and forearms, yet in these I ascribed the same to the strong chemical and lye soaps used. All were amenable to suitable therapentics. In the above cases I conld detect no cause, other than hamato-dyscrasia. There were no cases contiguous, no wounds which might have been the media of imbibing or localizing any specific virus.
The therapeutics were such as the cases indicated, principally ferruginous tonics, quinine added, and alkaline ablutions to counteract the intolerable itching or pruritus. Arsenic was combined with the tonics, also ergotin was given to stimulate proper contractility of the cutaneous vessels. One lady, in her incipient development, was annoyed by persistent nausea, which I obviated by a small mercurial with soda. I had had a practicing physician boast to me some months prior, that he had quite a number of cases of erysipelas. If there was any resemblance at all in the cases, I am sure that my cases were not erysipelas. Urticaria has been a freyuently recurring ailment in my experience, and has always been susceptible of ready relief. I have not had any so severe as to approximate to the subject of this sketch.

I feel that the symptoms will sustain me in my denomination of the malady, an erysipelatous eczema, the latter characteristic of red eczema.

285 Camp, St.

\section{INTUBATION OF THE LARYNX.}

BY L. H. DUNNING, M.D., or south BIEND, INIs.

(') Dwyer's method of intubing the larynx for the cure of membranous croup being under trial, the following history of an unsuccessful case is written in order to swell the list of recorded cases from which, when large enough, we must draw our conclusions as to the efficacy of the method.

On March 20 I received a note from Dr. J. B. Green asking me to come immediately to Mishawaka to see a patient of his, and to bring my ('Dwyer instruments. On reaching the bedside of the patient I found a child $21 / 2$ years old laboring under membranous croup, which had been in progress for eighteen hours. There was marked cyanosis, labored breathing and great prostration. These conditions had been gradually developing for several hours, and had now reached the point of jeopardizing the life of the child. Dr. Green and I agreed that unless instrumental means afforded relief death would be the inevitable result of the attack. Therefore, in what we deemed the discharge of our duty, we proceeded to intube the larynx.

The patient being slightly under size, we selected a tube nodeled for a two years' old child. The usual method of introducing the tube was observed, and I found little difficulty in properly placing it in the larynx. The tube afforded marked relief, and when the thread was removed the relief as evidenced by the patient's appearance was striking. She now breathed easily, the cyanosis disappeared, and she passed into a quiet natural sleep. After watching the patient for half an hour, and observing that the tube caused but little local irritation, and that the patient continued to breathe and rest easily, we directed that she should take as nourishment only warm milk, and then left her to the care of efficient nurses.

March 22, 8:30 A.M.-Pulse 120, respiration 22, temperature normal. Patient had rested well during night. Had coughed considerably on taking milk or 